\title{
Kirche im Dienst der Publizistik
}

von Remi J. De Roo

Die hier folgenden Gedanken spiegeln die Erfahrung eines kanadischen Bischofs wider. Er lebt inmitten einer pluralistischen Gesellschaft in einem zweisprachigen Land, in dem die Massenkommunikationsmittel größtenteils kommerziellen Rücksichten unterliegen.

Meine Uberlegungen stimmen in der Linie mit dem Zweiten Vatikanum überein. Das Konzil rief uns ja u. a. folgende Wahrheiten ins Gedächtnis:

Wir sind ein Pilgervolk und haben keine bleibende Heimat hier.

Wir leben in einer Welt sozialer und kultureller (R)evolution, die an der Oberfläche für Bewegung sorgt, aber auch in die Tiefe reicht.

Wir sind Wanderer, die - vom Geist Gottes durch mannigfache Gaben getrieben in sehr unterschiedlicher kultureller Umwelt leben und gerufen sind, recht viele verschiedene Dienste zu leisten.

Wir sind ein Volk mit einer umfassenden Sendung zum Dienst, uns freigewollt unseren Menschenbrüdern zur Verfügung zu stellen, jedoch unter voller Achtung ihrer Freiheit.

Wir sind ein Volk, dessen wirklich maßgeblicher Einfluß auf die Welt darin besteht, der Wahrheit zu dienen, indem es die Frohbotschaft Christi verkündet.

Wir leben in einer "demokratischen" Umwelt, in der jene Werte vorrangig sind, die dem Menschen die höchste Entfaltung garantieren und die persönliche Verantwortung fördern, die Entwicklung des Sozialgewissens und den offenen Dialog mit einer Welt, die um ihrer selbst willen geliebt und geachtet wird, wie auch den Kampf um soziale Gerechtigkeit für alle als Grundvoraussetzung für den von uns allen ersehnten gesicherten Frieden, an dem aber die Entrechteten ständig stärker verzweifeln, die zwischen friedlicher und blutiger Revolution in die Entscheidung gedrängt sind.

Diese meine Uberlegungen wollen an die Ausführungen von Kardinal Gray anschließen, der gestern zu uns über die Kirche sprach, ${ }^{1}$ die sich der sozialen Kommunikationsmittel bediene, um das Evangelium weiterzutragen. Er führte aus, die Kirche müsse heute nicht nur gesehen und gehört werden als eine Kirche, die betet und predigt und lehrt, sondern vielmehr als die Kirche, die tätig wird.

Ich möchte nun in gar keiner Weise die Bedeutung all des Gesagten schmälern, ich möchte auch keine Rangfolgen aufstellen noch auch möchte ich versuchen, die „Evangelisierung" gegenüber der "Prä-Evangelisierung" nach Verdiensten abzuwägen.

Dr. theol. Remi J. De Roo ist Bischof von Victoria (Vancouver, Canada) und Vorsitzender der Publizistikkommission der kanadischen Bischofskonferenz. Der Beitrag geht auf einen Vortrag zurück, den der Bischof auf der Generalversammlung der UNDA (11.-17. Januar 1971) in New Orleans gehalten hat. (Vgl. Bericht "UNDA-Weltversammlung in New Orleans", Seite 128). 
Vielmehr geht es mir darum, unsere Gedanken vorwärts zu richten und einen klaren Blick in die Zukunft zu versuchen. Ich möchte also eine weitere Dimension hinzufügen.

So stelle ich folgende Frage: Soll die Kirche, die die sozialen Kommunikationsmittel "in Dienst" nimmt, nicht ebensosehr - und möglicherweise sogar noch mehr - der Welt der sozialen Kommunikationsmittel selbst „dienen“?

Was heißt für uns "dienen"?

Bedeutet es nicht vor allem, das wahre Wesen der Massenmedien zu erkennen, damit man so ihre wirklichen Möglichkeiten berücksichtigen kann?

Es gibt ja eine ganz neue Kultursprache (mit allem möglichen unterschiedlichen Begriffs-Vokabular, z. B. audio-visuell, elektronisch, symbolisch, mehr emotional als intellektuell, säkulär, Massenkultur usw.) Ubberwinden wir immer die Versuchung, die neuen Medien zu mißbrauchen, wenn wir ihnen von außen her Ziele aufzudrängen suchen, für die sie nicht geschaffen sind?

Sind wir in einer pluralistischen Gesellschaft, in einer modernen Kultur, die wir mehr und mehr als „säkular" zu bejahen bereit sind und die ihre Eigenständigkeit und ihr eigenes Gepräge hat, wirklich realistisch, wenn wir ausschließlich daran denken, „katholisch “-sektiererische Programme zu entwickeln?

Schenken wir dem tatsächlichen Funktions-Prozeß der Medien genügend Beachtung, der ihnen charakteristisch eignet und auf Hörer und Zuschauer vielleicht einen tieferen Einfluß ausübt als der „Programminhalt"? Wenn wir den Massenkommunikationsmitteln mehr unsere eigenen Ziele aufzuzwingen versuchen, als daß wir ihr Wesen achten, liegt darin nicht vielleicht eine sehr augenfällige Gefahr?

Wäre es nicht besser, mit allen redlichen Menschen zusammenzuarbeiten, die Wahrheit, Schönheit, Gerechtigkeit, Liebe und Frieden suchen? Wer wird ihnen helfen, Christus bei der Ausübung ihres Berufes zu finden?

Das Konzil lehnt es ab, falsche politische Systeme zu verurteilen. Es lehrte geradezu, daß Christus in allen Menschen zugegen ist, selbst in Atheisten, die mit lauterem Herzen nach Wahrheit und Gerechtigkeit suchen. ${ }^{2}$

$\overline{\text { Wir }}$ sollten uns heute selbst einmal fragen: Was ist denn eigentlich ein „religiöses Programm"? Welchen Eindruck machen solche Programme auf den Menschen von heute, welche Botschaft haben sie wirklich für ihn? Wo dringt die Frohbotschaft ein? Wo wird nur Kultur-Müll geboten? Heute, wo die Menschen nach einem „Lebenssinn" Ausschau halten?

Ist die Kirche wirklich wirksam anwesend unter jenen Menschen, deren Leben von den Medien geprägt wird: unter den Leuten vom Fach, unter Hörern und $\mathrm{Zu}$ schauern?

Würde die Kirche nicht gewinnen durch stärkeres Zusammenarbeiten mit allen, die ihre Talente als redliche Publizisten in den Dienst der Gesellschaft stellen? Ist es realistisch, weiterhin eigene Mediensysteme auszubauen, die immer mehr Geld verschlingen, und damit eine Konkurrenzpolitik zu den "säkularen" Medien zu betreiben? Es kann durchaus sein, daß unsere kostspieligen Institutionen öfter den Anschein von Macht und Rivalität erwecken als den Eindruck uneigennützigen Dienstes. Manchmal dienen sie dazu, den „status quo“ in Gesellschaften zu erhalten, 
in denen die soziale Ungerechtigkeit herrscht. Sind sie denn dann wirklich frei, den Armen die Frohbotschaft zu künden? Sprechen sie wirklich für die, die keine Stimme haben? Woher kommt denn eigentlich das Geld, um den ungeheuer aufwendigen Weiterbestand dieser Unternehmen sicherzustellen?

Dienen könnte heißen, einen Dialog zu akzeptieren, einen freien, liebevollen Dialog, der die Meinungen des anderen respektiert, eher in einer Atmosphäre gemeinsamer Wahrheitssuche als mit bestimmender Autorität. Auf diese Weise würde man auf den Geist Gottes hören, der sich selbst in der christlichen Anwort oder im gemeinsamen Empfinden aller Glieder des Gottesvolkes, auch der „kleinen Leute“, erschließt.

Dienen könnte heißen, die Zeichen der Zeit zu erkennen und in ihnen die Gegenwart Gottes zu sehen, der in unser aller Mitte lebt und wirkt und sich in den Ereignissen unserer Zeitgeschichte kundtut (modernes Bewußtsein, „Bewußtwerdung“, Entwicklungs- und Befreiungstheologie)?

Dienen könnte auch heißen, auf das zu hören, was die Welt uns durch die Medien sagt. Dulden wir die Stimme der Massenmedien nur, derweil wir nur auf eine Gelegenheit warten, sie zu „bekehren“? Oder anerkennen wir, daß die Kirche selbst ohne sie ebensoviel ärmer wäre und ihrer Sendung nicht voll entsprechen könnte, weil sie sich in der Welt in einen (r)evolutionären Ablauf hinein „inkarniert" findet?

Sicherlich sollte die Stimme der Kirche in der Gesellschaft, in der Schöpfung gehört werden. Aber sollte die Stimme der Schöpfung und der menschlichen Gesellschaft, die nach „ihrer" Wahrheit und nach "der" Wahrheit ausschaut, nicht auch in und durch die Kirche gehört werden?

Dienen könnte heißen, die unterschiedlichen Ausdrucksweisen der Wahrheit zu akzeptieren, ohne daß man wünscht, sie nur in die gewohnten Schablonen einer sich ändernden (sich auflösenden) Kultur zu pressen? Vielleicht steckt hinter unserem Eifer für eine genaue und wohlbehütete "Rechtgläubigkeit" bisweilen mangelnder Glaube an den Geist Gottes, der allen Gliedern Christi Leben schenkt? Wie bequem ist es doch, unseren Brüdern zu mißtrauen, die die Frohbotschaft im elektronischen Zeitalter in eine neue Sprache übersetzen wollen: in moderne Musik, in Dichtung, Kunst, Psychologie, Philosophie, Soziologie, Theologie usw.

Was den ökumenischen Dienst angeht, so sind die Grundsätze klar und ist die Richtung gewiesen. Zeigt unser Tun denselben Geist? Das Zweite Vatikanum anerkannte eine "Hierarchie" der Wahrheiten in der Verkündigung. Wenn auch alle wahr seien, so seien sie doch nicht alle gleich wichtig. ${ }^{3}$

Sind wir bereit, mit den anderen Kirchen, die als Heilsgemeinschaften anerkannt sind, zusammenzugehen, um mit gemeinsamer Stimme einer Welt, die nach Einheit und Brüderlichkeit sucht, die grundlegenden Wahrheiten zu künden? Sind wir bereit, in Fragen der sozialen Gerechtigkeit gemeinsame Sache zu machen?

Das Konzil riet, Úberschneidungen von Arbeitsgebieten und Rivalität zu vermeiden. Wir sollten mit unseren christlichen Brüdern auf allen Gebieten, wo Grundsatzfragen und Gewissensgründe es nicht verbieten, als Mannschaft zusammenarbeiten. Setzen unsere Entwicklungspläne auf dem Gebiet der Massenkommunikationsmittel tatsächlich die ökumenische Zusammenarbeit in Rechnung? Zu welchen Opfern sind wir bereit, welche personellen oder finanziellen Verpflichtungen nehmen wir 
um der gemeinsamen Sache willen in Kauf? Sollen wir weiterhin nur unsere eigenen Gläubigen ansprechen, oder sollen wir zu allen Menschen sprechen, die unsere Brüder sind?

Mißverstehen Sie mich bitte nicht! Ich will keineswegs die großartige Arbeit so vieler Wegbereiter kritisieren, die den Kampf vor uns aufnahmen oder noch immer unter so schwierigen Umständen weiterkämpfen. Es gibt auch Propheten von gestern, auf die wir nicht hören wollten und denen wir heute doch gestehen müssen, daß sie recht hatten. Manche von ihnen bauten die Kirche irgendwo in einem vergessenen Winkel fast ohne Hilfe allein auf. Ich achte und bewundere sie von ganzem Herzen.

Aber die Welt schreitet vorwärts und mit ihr die Menschen. Die das Steuer der Kirche lenken, brauchen alles Licht der heute nur möglichen Einsicht. Die Kirche braucht einen echten Dialog, einen Meinungsaustausch in beiden Richtungen (twoway communication), informierte und verantwortete öffentliche Meinung. Darum stelle ich diese Fragen. Und darum erbitte ich Ihre Antwort und Thre Kritik.

Zum Dienst der „christlichen Präsenz" in Hörfunk und Sehfunk biete ich hier zum Schluß einige praktische Vorschläge an. Christliche Präsenz kann sich in zwei Richtungen entfalten. Hier nur einige Möglichkeiten, die wir überprüfen könnten:

\section{In der Fachwelt der Kommunikatoren:}

Wir könnten versuchen, uns zu christlicher Besinnung zu treffen.

Wir könnten offene und freundschaftliche Beziehungen zu Fachleuten aufnehmen und pflegen.

Wir könnten unseren Rat zur Verfügung stellen.

Wir könnten gemeinsam überlegen, wie Gottesdienstformen sich zeitgemäß den Sondererfordernissen der Massenmedien entsprechend anpassen ließen.

Wir könnten junge Leute ermutigen, die Medienberufe als einen Dienst an der Menschheit zu begreifen und zu ergreifen.

\section{In der Rezipienten-Situation der Hörer und Zuschauer:}

Wir könnten einen Dialog zwischen Produzenten und Empfängern der Programme organisieren.

Wir könnten die Empfänger, die Konsumenten, daran gewöhnen, jene Produzenten zu unterstützen und zu ermuntern, die nach ihrem Gewissen handeln.

Wir könnten gemeinsam unsere pastorale Auffassung überprüfen und dabei den neuen Menschen in einer durch die Medien geschaffenen neuen Gesellschaft berücksichtigen.

Wir könnten der Kirche insgesamt zum Bewußtsein über die Bedeutung der Information in, durch und über die Kirche verhelfen. Information, eine unterrichtete öffentliche Meinung und verantwortliches Mitwirken gehen Hand in Hand und sind, damit eine christliche Gemeinschaft wirklich erwachsen wird, unentbehrlich.

Wir könnten in Personen und Mittel investieren, um dadurch bei den Menschen kritischen Sinn und christliches Gewissen zu wecken. 


\section{Anmerkungen:}

1. Gordon Kardinal Gray, Erzbischof von St. Andrews und Edinburgh, Mitglied der Päpstlichen Kommission für die Soziale Kommunikation, hielt auf dem Unda-Weltkongreß in New Orleans am 12. Januar 1971 eine längere Ansprache über die Aufgaben und Möglichkeiten der Kirche im Bereich der publizistischen Medien, die den Teilnehmern vervielfältigt vorlag.

2. Vgl. die Konzilskonstitution "Die Kirche in der Welt von heute", in: Karl Rahner und Herbert Vorgrimler: Kleines Konzilskompendium, Freiburg 1966, S. 466 bis 470 (Nr. 21 und 22).

3. Vgl. Konzilsdekret „Ưber den Okumenismus“, in: Karl Rahner und Herbert Vorgrimler, a.a.O., S. 229 und 230 (Nr. 11).

\section{S UM M A R Y}

The Canadian Bishop de Roo in his speech at the UNDA General Assembly in New Orleans asks if the church as well as putting the means of social communication at her "service" should not "serve" the world of social communications. This "service" would mean e. g. to recognize the real nature of the social communications media in order to respect their true potential; to accept dialogue; to recognize the signs of the times and discern in them the presence of god; to listen to what the world is saying to us through the media; to accept the various expressions of truth without wishing to channel them only along the familiar lines of a changing culture.

\section{R E S U M EN}

El Obispo canadiense de Roo, en su discurso a la Asamblea General de la UNDA celebrado en Nueva Orleans, pregunta si la Iglesia debe poner los Medios de Comunicación Social a su "servicio“ o si no debería también „servir“ al mundo de las Comunicaciones Sociales. Este „servicio“ significaría, por ejemplo, reconocer la naturaleza real de los Medios de Comunicación Social para respetar sus verdaderos potenciales; aceptar el diálogo; reconocer los signos de los tiempos y discernir en ellos la presencia de Dios; escuchar lo que nos dice el mundo a través de los medios de comunicación; aceptar las diferentes expresiones de la verdad sin desear canalizarlas solamente junto con las líneas familiares de una cultura en contínuos cambios. 